\section{Cahiers de Narratologie}

Analyse et théorie narratives

$34 \mid 2018$

Les avatars du chapitre en bande dessinée

\title{
Les deux Ligurie. Francesco Biamonti sur les traces de Jean Giono
}

\section{Matteo Grassano}

\section{(2) OpenEdition}

\section{Journals}

\section{Electronic version}

URL: http://journals.openedition.org/narratologie/8930

DOI: 10.4000/narratologie.8930

ISSN: $1765-307 X$

Publisher

LIRCES

\section{Electronic reference}

Matteo Grassano, «Les deux Ligurie. Francesco Biamonti sur les traces de Jean Giono », Cahiers de Narratologie [Online], 34 | 2018, Online since 21 December 2018, connection on 22 April 2019. URL : http://journals.openedition.org/narratologie/8930; DOI : 10.4000/narratologie.8930

This text was automatically generated on 22 April 2019

Article L.111-1 du Code de la propriété intellectuelle. 


\title{
Les deux Ligurie. Francesco Biamonti sur les traces de Jean Giono
}

\author{
Matteo Grassano
}

1 Francesco Biamonti (1928-2001) est désormais entré dans le canon littéraire des écrivains majeurs italiens de la fin du XXème siècle. Né à San Biagio della Cima, un petit village dans l'arrière-pays de Vintimille, Biamonti a entièrement consacré sa vie à la littérature et à l'écriture. Toutefois, il n'a publié son premier roman, L'angelo di Avrigue (1983), qu'à l'âge de cinquante-cinq ans, grâce à l'intervention de Nico Orengo et d'Italo Calvino ${ }^{1}$. Il est aussi l'auteur de Vento largo (1991), d'Attesa sul mare (1994) et de Le parole la notte (1998). Un dernier roman inachevé, intitulé Il silenzio, est paru posthume en $2003^{2}$.

Dans ces romans, dont les histoires se déroulent dans l'arrière-pays ligurien près de la frontière franco-italienne, l'image de la France est omniprésente, que ce soit à travers les voyages, les souvenirs ou les désirs des protagonistes. Ces éléments s'expliquent en substance par la francophilie de l'auteur. Il faut rappeler que la formation de Biamonti doit beaucoup à la culture française, comme en attestent les volumes de sa bibliothèque. Pendant les années cinquante et soixante, Biamonti se plongea dans l'étude des ouvrages existentialistes et phénoménologiques de Sartre, Camus, Merleau-Ponty. En même temps, dès sa jeunesse, il fut un grand admirateur de Baudelaire et de Cézanne, qu'il considérait comme les pères fondateurs de la poésie et de l'art modernes.

En ce qui concerne les romanciers français, l'écrivain ligurien a toujours été un lecteur attentif et passionné de l'œuvre de Jean Giono. Presque tous les romans gioniens sont conservés dans sa maison à San Biagio della Cima. Si l'on relit ses écrits critiques et ses interviews, il est possible de découvrir plusieurs références explicites à l'écrivain de Manosque. En 1994, décrivant une journée en Provence, Biamonti écrivait, par exemple : « Je suis au cœur d'une France fascinante. Giono a situé ses romans dans le plateau. C'était un grand écrivain qui marchait à pied sur des routes pleines de pierres et de soleil. "Je dois encore explorer la haie devant chez moi", disait-il à ceux qui l'invitaient à faire de grands voyages $»^{3}$. 


\section{de celui de Valéry :}

Si je pense à la littérature française, à ma dette envers l'art de France, je vois un pays baigné par la mer, des collines érodées par la lumière, surmontées par un azur désincorporant et transformant la matière. C'est un pays qui va des plateaux de Provence, où encore transhument les personnages de Giono, jusqu'aux sables de Sète, où Valéry a scruté le mystère des tombeaux sur la mer et a peu à peu composé une autre mer, pétrie de diamants extrêmes, labourés par le vent et par le soleil ; c'est une image de la vie et de la mort ${ }^{4}$.

Le Plateau de Valensole constitue d'ailleurs le décor du dixième chapitre du roman Vento largo, qui est un véritable hommage à Giono. Suite à la disparition de Sabèl, une jeune femme originaire d'un village de l'arrière-pays ligurien, Varì, le protagoniste du livre, part à sa recherche « dans les champs de lavande et de vent, dans ces lieux-dits dont elle lui avait souvent parlé $»^{5}$. Nous sommes au mois de juillet et la cueillette de la lavande est désormais commencée. L'homme traverse une partie du Plateau et interroge les équipes de ramasseuses qu'il croise sur son chemin. Il va à Saint-Martin-de-Brômes, à Riez, à Moustier-Sainte-Marie. Biamonti décrit ainsi le village de Saint-Martin-de-Brômes et le paysage environnant :

C'était le règne du vent. Un mistral brisé sur sa fin secouait encore le village: quelques très vieilles maisons, avec de grands vestibules et, sur l'architrave des portes, la date ; trois ruelles et un clocher en forme de pyramide aux trois hautes baies ovales. [...]

Il sortit du village : le plateau tout entier était un radeau dans le ciel. Un autre vent s'était levé : la brise de la montagne de Lure. Le désert des lavandes - une terre de soleil - était maintenant froid et constellét.

Dans cette description, Biamonti se souvient clairement de l'incipit gionien de Colline (1929) : « Un débris de hameau, à mi-chemin entre la plaine où ronfle la vie tumultueuse des batteuses à vapeur et le grand désert lavandier, le pays du vent, à l'ombre froide des monts de Lure $»^{7}$. Varì ne trouve pas Sabèl sur le Plateau de Valensole, car elle s'est réfugiée sur l'île de Saint-Honorat, l'une des Îles de Lérins, afin de travailler dans les petits champs de lavande et dans les vignobles des moines. Au début du seizième chapitre, qui a pour cadre l'île de Saint-Honorat, on retrouve une autre référence à Giono : «Elle [Sabèl] écoutait le chant de complies “... que ma joie demeure..." Il passait avec une douceur majestueuse dans l'azur poreux du crépuscule $»^{8}$.

Il importe encore de souligner que l'espace narratif du dernier livre de Biamonti, Le parole la notte, s'ouvre derechef sur une partie du territoire provençal par la médiation de Giono. Dans ce cas il ne s'agit plus du Plateau de Valensole mais du Plan de Canjuers. Dans le dernier chapitre du roman, les personnages principaux partent de l'arrière-pays ligurien pour rejoindre Bargème, un petit village aux pieds de la montagne de Brouis, où le vieil officiel français Corbières a disposé que ses cendres soient dispersées. Au retour, le groupe s'arrête dîner dans un autre village, Bargemon, où s'achève l'histoire :

Ils prirent une autre route pour revenir. Ils passèrent par le camp militaire de Canjuers : haut plateau doré et gris, avec au milieu un village abandonné et protégé par des barbelés. Puis la route descendait sur Bargemon, ourlée de genévriers. Ils s'arrêtèrent sur une place entourée de vieilles ruelles. En son centre se dressait, à l'ombre des micocouliers, une fontaine envahie par la mousse. Un chien de chasse dormait sur un banc. ${ }^{9}$

8 Ce village est aussi le point d'arrivée de l'Itinéraire de Manosque à Bargemon (1963) de Giono que Biamonti connaissait sans aucun doute : «Enfin, une vapeur qui tremble à l'horizon 
signale Bargemon, et on descend, tout étonné, dans un village du Moyen Age qui paraît, après ce qu'on a vu, une anticipation de l'an 2000. D'ici, déjà, par-dessus la vallée où dorment des saules opulents, on peut entendre les rumeurs de la Nationale 7 et même de la côte d'Azur $»^{10}$. En outre, le nom de l'officiel Corbières rappelle celui d'un village de la vallée de la Durance, où s'accomplit la fabuleuse éducation sentimentale de Jean le Bleu.

Toutefois, la présence de Giono dans l'œuvre biamontienne ne saurait se borner à l'intertextualité ponctuelle impliquée par ces citations. Afin de comprendre pleinement l'influence de l'auteur de Manosque sur les romans de Biamonti et sur sa poétique, il faut en effet aborder certains sujets caractérisant les œuvres de ces deux écrivains. Tout d'abord, on peut considérer que Biamonti avait lu de manière intégrale et approfondie l'ouvrage critique La Provence de Giono de Jacques Chabot, paru en 1991 : un exemplaire de cet essai se trouve dans la bibliothèque de l'auteur ligurien et montre plusieurs marques de lecture de bout en bout. C'est justement la façon dont Giono a su laisser parler aussi bien la réalité que le rêve de son territoire qui a préalablement frappé l'imagination de Biamonti et qui a fini par l'influencer. Si l'auteur de Manosque a écrit que «le monde inventé n'a pas effacé le monde réel : il s'est superposé $»^{11}$, Biamonti pour sa part répétait souvent que «c'est notre destin humain d'habiter le monde, mais c'est aussi notre destin humain d'en rêver un autre $\|^{12}$. Ce jeu entre réalité et fiction apparaît à travers la représentation du monde traditionnel de leurs régions, proposée par les deux écrivains.

Comme Chabot l'écrit dans son ouvrage, tous les villages provençaux gioniens «sont des villages morts ou en train de mourir : la Provence de Giono représente la fin d'un monde ; il est un des derniers témoins de la civilisation paysanne qui s'établit sur le pourtour de la Méditerranée du néolithique à la Révolution française. Vers 1930 l'agonie s'achève $»^{13}$. On pourrait utiliser des mots équivalents pour décrire la Ligurie biamontienne, dont l'auteur dépeint toujours l'arrière-pays dans un état d'abandon, avec les oliveraies malades ou délaissées, les terrasses en ruine, les villages morts où ne restent désormais que des personnes âgées. Biamonti a défini lui-même son deuxième livre « un chant d'adieu pour une Ligurie qui entre dans l'Érèbe $»^{14}$, dont le protagoniste, Varì, est « le dernier témoin d'une vie qui s'en allait $»^{15}$.

La vie qui s'en va est celle de l'ancien monde traditionnel, de la civilisation paysanne, de la « civilisation de l'olivier » :

C'est la civilisation de l'olivier. Une civilisation magnifique. Il y a deux mille ans, les Grecs nous apprirent à greffer l'olivier sur le chêne vert. Aujourd'hui, après deux mille ans, cette civilisation est morte et ses communautés sont mortes avec elle. C'était une société très douce. Quiconque pouvait bien y vivre avec un peu plus de cinq cents oliviers. Maintenant, cela n'est plus possible: les oliveraies restantes sont presque toutes abandonnées, les gens d'aujourd'hui sont seuls, dénaturés. On survit avec les floricultures, les serres ont remplacé les oliveraies et les plus malchanceux sont obligés d'être serveurs à Monaco. La civilisation de l'olivier est morte, mais aucune autre ne l'a remplacée. ${ }^{16}$

12 C'est au fond la tragédie de la civilisation paysanne, qui entraîne la perte d'un savoir et d'un mode de vie millénaires. Connaissant la détresse et la fatigue du monde rural, Biamonti n'idéalise pas la vie traditionnelle (ses personnages maudissent toujours leur propre enfance, où il n'y avait rien), mais force lui est de reconnaître que cette civilisation avait permis une vie simple et harmonieuse, délivrée de besoins superflus, dès que l'homme s'était accordé « au cosmos comme une pierre » ${ }^{17}$. L'olivier, qui « n'est même pas un arbre mais un rêve d'arbre $»^{18}$, est sans aucun doute le symbole de cette civilisation : son histoire permet un retour aux origines et pose ce monde dans un cadre 
méditerranéen. En effet, comme l'a écrit Fernand Braudel, « la Méditerranée court ainsi du premier olivier atteint quand on vient du nord aux premières palmeraies compactes qui surgissent avec le désert $»^{19}$. La Ligurie est donc pour l'écrivain de San Biagio della Cima « la zone la plus au nord de l'oliveraie et [...] de la palmeraie méditerranéennes : c'était, pour ainsi dire, le bord d'une civilisation très ancienne qui s'était mêlée au territoire au point d'apparaître comme spontanée $»^{20}$.

L'expression "civilisation de l'olivier » est empruntée, avec une petite modification, à Giono: "Est-ce qu'on peut imaginer une civilisation de la nèfle! Nous sommes de la civilisation de l'olive, nous autres. Nous aimons l'huile forte, l'huile verte $»^{21}$. D'ailleurs, Biamonti connaissait presque par cœur les écrits gioniens où l'on représente la culture de l'olivier, tels qu'Arcadie... Arcadie... (1953), Manosque-des-Plateaux (1930) ou Le poème de l'olive (1931). À propos de ce dernier, dans un article de 1991, l'écrivain italien a évoqué la

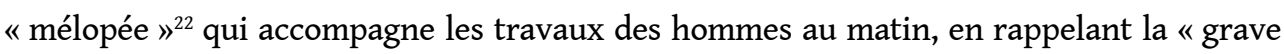
mélopée d'un chant qui vient de l'enfer ${ }^{23} \mathrm{du}$ texte gionien. On peut même ajouter que tous les protagonistes des romans biamontiens possèdent une oliveraie et que Biamonti décrit à maintes reprises, souvent de manière minutieuse et technique, les différents processus liés à l'exploitation de l'olivier.

Cependant, si les crises ligurienne et provençale de la « civilisation de l'olive » évoquées par Giono et par Biamonti ont des caractéristiques similaires, il faut remarquer qu'elles ne se situent pas sur le même plan temporel. Biamonti ne peut qu'éprouver une grande fascination pour l'œuvre de l'auteur de Manosque, dès lors qu'il se rend compte que les transformations sociales, économiques et territoriales subies par la Provence depuis la deuxième moitié du XIXème siècle et dénoncées par Giono avec une force extraordinaire à partir des années vingt sont également celles qui ont bouleversé sa Ligurie, cinquante ans plus tard ${ }^{24}$.

Originaire d'une famille paysanne, Biamonti connaissait bien le monde traditionnel de l'arrière-pays ligurien, encore intègre lorsqu'il était enfant, même si pendant les années vingt et trente on y voyait déjà les premiers signes de changements, tels que la naissance du tourisme sur la Riviera et la prolifération des serres et des floricultures remplaçant les oliveraies. Toutefois, c'est pendant les années cinquante et soixante que le Ponant ligurien a vécu sa révolution la plus frappante, à cause du développement du tourisme de masse et de la spéculation immobilière (on pense ici au livre homonyme d'Italo Calvino, paru en 1963). Le passage d'une économie agricole à une économie touristique n'a pas seulement favorisé de plus en plus l'urbanisation de la côte au détriment de l'arrièrepays, mais il s'est traduit également par un bétonnage incontrôlé du territoire côtier. À ce propos, l'historien italien Bruno Gabrielli a parlé d'une véritable "dilapidation du territoire ", en soulignant que «le modèle de développement a été celui de résidences secondaires, qui a produit un phénomène de conurbation côtière dont le résultat est la soudure complète, sans solution de continuité, de longs segments côtiers $»^{25}$. San Biagio della Cima se trouvant dans une zone intermédiaire hybride entre la côte et l'arrière-pays montagneux, Biamonti a vu de près cette transformation radicale. Ses romans et ses écrits montrent son aversion pour une modernité destructrice, qui a brutalement coupé les ponts avec le passé :

Je crois que le devoir d'une civilisation réelle est de maintenir une continuité des choses et de ne pas rompre brutalement avec le passé ; tout étant voué à mourir, que cela meure au moins avec douceur. Ici, au contraire, tout meurt brutalement: on coule du béton, on élève des bâtiments, on détruit la mer, on assiège les 
cimetières de maisons, on détruit les palmeraies... des destructions parfaitement inutiles! ${ }^{26}$ deux Provence, et écrit: "Avec un fanatisme intransigeant, altier, sans concessions, il partage en deux la carte géographique de la Provence, et il ne reconnait que celle d'en haut : il ne légitime pas celle d'en bas $\aleph^{27}$. D'un côté, il y a donc le pays d'en haut, avec ses montagnes et ses hauts plateaux, la Provence pudique et secrète, la Provence fière et morale qui, bien que dans un état d'abandon, conserve encore l'esprit de l'ancienne civilisation paysanne. D'un autre côté, il y a le pays d'en bas, la Provence sans fierté et vulgaire: "Aborder l'Italie par la mer, c'était l'aborder écorché vif. Il fallait longer d'abord toute cette côte d'Azur si vulgaire, et suivre ensuite, tout autour du golfe de Gênes, les rivières du Ponant et du Levant. Cela faisait beaucoup trop de papier de verre, de râpe à fromage, de kilomètres de femmes à poil en train de sécher sur le sable ${ }^{28}$. C'est justement cette Provence-là, exhibitionniste, racoleuse, vendue, faite pour les touristes, que Giono répudie :

Comme tout le monde, je connais ce qu'on appelle bêtement la Côte d'Azur. Quel est le chef de rayon qui a inventé cette appellation? Si on le connaît qu'on le décore : il avait le génie de la médiocrité. Notre pays est en toute saison traversé par le fleuve de Parisiens, de Belges, d'Anglais et d'Esquimaux qui va se jeter dans la Méditerranée. C'est un Mississipi qui déborde en une Louisiane de marais, de crocodiles et de crapauds-buffles. Sur la côte, on débite l'azur comme un thon. Pas une dactylo d'Anvers, de Roubaix ou de Glasgow qui ne rêve de faire sa cocotte et sa grande coquette en en bouffant une tranche. On arrive et on se fout à poil.

Rien de commun avec le vrai pays ${ }^{29}$.

17 La Ligurie est divisée en deux également aux yeux de l'auteur de San Biagio della Cima. Dans Le parole la notte, le protagoniste a cette pensée : « Il y a deux Ligurie [...] Une côtière, avec des trafics de drogue, envahie et massacrée par les constructions, et une autre montagnarde, une sorte de Castille encore austère; je suis à la frontière des deux $»^{30}$. Biamonti a souvent dénoncé avec des mots assez proches de ceux de Giono ce double visage de sa région, en prenant toujours en compte la dualité destruction-abandon :

Or, lorsque j'entends n'importe quel écrivain ou j'interpelle n'importe quel ami qui vient ici, on me dit: Qu'est-ce que cette Ligurie, cette ligne malodorante d'essence avec une jungle de maisons qui l'entourent? Une mer qu'on ne voit même plus, dont on n'entend plus le souffle, et un arrière-pays abandonné ? Nous faisons face à deux types de dégradations : l'une est une intensité cancéreuse de vie et l'autre un lent abandon de la vie. [...]

La grande destruction s'est produite durant les années soixante et soixante-dix, mais elle s'est produite partout dans le monde, en France, en Italie, sur la Côte d'Azur, où il n'y a plus rien qui ne soit pas faux, elle s'est produite au plus haut niveau à Monaco; et je ne vais donc pas imputer aux hommes de Bordighera la faute d'une agitation frénétique qui a touché le monde entier ${ }^{31}$.

Toutefois, si la côte est désormais totalement perdue et est devenue le symbole d'une modernité immorale et brutale, une partie de l'arrière-pays garde encore les traces de l'ancienne civilisation et du paysage originaire. Il y a une Ligurie qui, bien qu'en ruine, a été sauvegardée et est encore authentique: c'est - nous dit Biamonti- la Ligurie rocheuse, inaccessible et verticale, et il regrette que toute la Ligurie ne soit pas ainsi verticale, de manière à empêcher la curée des spéculateurs.

Gaston Bachelard, l'un des philosophes français les plus lus et appréciés par Biamonti, écrivait que "toute verticalisation est valorisation $»^{32}$. Pour cette raison, la verticalité n'est pas seulement une caractéristique physique du territoire, mais elle est aussi une 
valeur. L'affection et le regard de l'écrivain de San Biagio della Cima s'attachent à la Ligurie qui, comme le pays d'en haut de Giono, est une "Castille encore austère ", où «l'ancestralité et la sacralité de la vie sont encore des valeurs quotidiennes " ${ }^{33}$. Dans ses romans, aussi bien à travers l'évocation de l'agonie présente qu'à travers l'image portée par la mémoire, Biamonti représente donc la vie d'une Ligurie traditionnelle; il laisse souvent la parole à ses paysans - vieux et sans désillusions, qui s'obstinent à cultiver les oliviers et les vignes -, à leur culture populaire, à leurs chansons. Toutefois, il n'y a jamais chez Biamonti, à différence de Giono, la tentative ou l'espoir de reconstruire le monde ancien. Le mouvement historique est inexorable, et toute chose est destinée à mourir. Si les protagonistes biamontiens souffrent de ce changement parce que, comme le disait Baudelaire, « le cœur d'une ville change plus vite que le cœur d'un mortel $~_{34}$, ils n'ont pas la force ni la conviction de poursuivre le chemin tracé par leurs ancêtres. Leur effort est plutôt celui de conserver la vérité éthique et existentielle la plus profonde de leurs origines et, par conséquent, de se battre pour rester humains dans un monde de plus en plus inhumain.

En conclusion, la comparaison entre l'œuvre de Biamonti et celle de Giono est tout d'abord induite, comme on l'a démontré, par la façon dont les deux écrivains ont vécu la transformation de leur territoire sous la marche du soi-disant progrès, et dont ils ont essayé de faire entendre la voix d'une civilisation paysanne menacée de disparaître ${ }^{35}$. Dans cette perspective, l'œuvre de Giono aura constitué un point de repère constant pour Biamonti, qui a trouvé dans l'auteur de Manosque un précurseur de sa lecture du monde traditionnel, capable de marcher d'un pas attentif et poétique parmi les ruines de l'Histoire.

\section{BIBLIOGRAPHY}

Bachelard Gaston, L'air et les songes. Essai sur l'imagination du mouvement, Paris, José Corti, 1992.

Baudelaire Charles, Les Fleurs du Mal, édition de 1861, texte présenté, établi et annoté par Claude Pichois, Paris, Gallimard, 1996.

Biamonti Francesco, « Non c'è più pace tra gli ulivi », interview réalisée par Antonio Troiano, in Corriere della Sera, 31 mars 1991.

Id., " Antico ! Così lo chiama Montale. Il Mediterraneo è antico e tragico ", in In Europa, 27, 3 août 1991 ; puis in Id., Scritti e parlati, op. cit., pp. 121-122.

Id., Vent largue, trad. Bernard Simeone, Lagrasse, Verdier, 1993.

Id., « Una giornata azzurra », in La Repubblica, 31 juillet 1994 ; puis in Id., Scritti e parlati, op. cit., pp. 125-128.

Id., « Biamonti, l'anti-estate », interview réalisée par Antonella Viale, in Il Secolo XIX, 4 août 1996.

Id., « [sans titre] », Corrado Ramella (dir.), in Il Gabbiano, 1997, pp. 1-3.

Id., « Un'antica luce per il 2000 », in Il Gazzettino, 9 septembre 1998, p. 21 ; puis in Id., Scritti e parlati, op. cit., pp. 31-33. 
Id., « [sans titre] », in Mario Novaro tra poesia e cultura, Actes de Colloque, Firenze, Le Monnier, 1998, pp. 197-198; puis in Id., Scritti e parlati, op. cit., pp. 44-45.

Id., Les paroles la nuit, trad. François Maspero, Paris, Seuil, 1999.

Id., « Se penso alla letteratura francese », in Eutropia, 1, mai 2001, p. 75.

Id., Scritti e parlati, textes réunis et présentés par Gian Luca Picconi e Federica Cappelletti, préface de Sergio Givone, Torino, Einaudi, 2008.

Braudel Fernand, La Méditerranée. L'espace et l'histoire, Paris, Flammarion, 1977.

Calvino, Italo, Lettre à F. Biamonti (Roma, 21 octobre 1981), in Id., Lettere 1940-1985, Luca Baranelli (dir.), introduction de Claudio Milanini, Milano, Mondadori, 2000, pp. 1456-1457.

Chabot Jacques, La Provence de Giono, Aix-en-Provence, Édisud, 1991.

Gabrielli Bruno, « La dilapidazione del territorio », in La Liguria, Antonio Gibelli et Paride Rugafiori (dir.), Torino, Einaudi, 1994, pp. 779-803.

Giono Jean, Colline, Paris, Grasset, s.d. [1929].

Id., Noé, Paris, Éditions de la Table Ronde, 1947.

Id., Voyage en Italie, Paris, Gallimard, 1953.

Id., Manosque-des Plateaux, suivi de Poème de l'olive, Paris, Gallimard, 1998.

Id., Arcadie... Arcadie..., précédé de La pierre, Gallimard, Paris 2001.

Id., Provence, textes réunis et présentés par Henri Godart, Paris, Gallimard, 1995.

Grassano Matteo, « "D’uno lengo vieio que se vôl pas cala...”. Francesco Biamonti e il mito provenzale », in Italica, 94, 3 (2017), pp. 546-562.

Malraux André, Les noyers de l'Altenburg, Paris, Gallimard, 1948.

Marengo Marina, «Sguardi letterari sulle "terre di frontiera” : le rappresentazioni delle Alpi sudoccidentali nelle opere di Francesco Biamonti e Jean Giono, in Intemelion », 22 (2016), pp. 89-104.

Orengo Nico, « La cura per una mimosa fece scoprire Biamonti », in La Stampa, 10 juillet 2003, p. 12.

\section{NOTES}

1. Cf. I. Calvino, lettre à F. Biamonti (Roma, 21 octobre 1981), in Id., Lettere 1940-1985, Luca Baranelli (dir.), introduction de Claudio Milanini, Milano, Mondadori, 2000, pp. 1456-1457 ; et N. Orengo, «La cura per una mimosa fece scoprire Biamonti », in La Stampa, 10 juillet 2003, p. 12.

2. Tous les romans de Biamonti ont été traduits en français: L'ange d'Avrigue, trad. Philippe Renard, Lagrasse, Verdier, 1990 ; Vent largue, trad. Bernard Simeone, Lagrasse, Verdier, 1993 ; Attente sur la mer, trad. François Maspero, Paris, Seuil, 1996; Les paroles la nuit, trad. François Maspero, Paris, Seuil, 1999 ; Le silence, trad. Carole Walter, Lagrasse, Verdier, 2005.

3. «Sono nel cuore di una Francia affascinante. Nell'altopiano Giono ha ambientato i suoi romanzi. Grande scrittore che marciava a piedi sulle strade piene di pietre e di sole. "Devo ancora esplorare la siepe davanti a casa", diceva a chi lo invitava ai grandi viaggi », F. Biamonti, "Una giornata azzurra ", in La Repubblica, 31 juillet 1994 ; puis in Id., Scritti e parlati, textes réunis et présentés par Gian Luca Picconi e Federica Cappelletti, préface de Sergio Givone, Torino, Einaudi, 
2008, p. 127. Sauf pour les extraits de romans, tous les textes de Biamonti et d'autres auteurs italiens ont été traduits par nos soins.

4. «Se penso alla letteratura francese, al mio debito verso l'arte di Francia, mi si forma un paese bagnato dal mare, di colline erose dalla luce, cui sovrasta un azzurro che scorpora la materia e la trasforma. È un paese che va dagli altopiani di Provenza, dove ancora transumano i personaggi di Giono, alle sabbie di Sète dove Valéry ha scrutato il mistero delle tombe sul mare e a poco a poco ha composto un altro mare, intriso di diamanti estremi, lavorati dal vento e dal sole, che è un'immagine del vivere e del morire ", Id., «Se penso alla letteratura francese ", in Eutropia, 1, mai 2001, p. 75.

5. Id., Vent largue, op. cit., p. 63.

6. Ibidem, pp. 64-65.

7. J. Giono, Colline, Paris, Grasset, s.d. [1929], pp. 9-10. Nous citons l'édition que Biamonti possédait et qu'il avait annotée.

8. F. Biamonti, Vent largue, op. cit., p. 103.

9. Id., Les paroles la nuit, op. cit., p. 219.

10. J. Giono, Provence, textes réunis et présentés par Henri Godart, Paris, Gallimard, 1995, p. 257.

11. J. Chabot, La Provence de Giono, Aix-en-Provence, Édisud, 1991, p. 14

12. F. Biamonti, «Un'antica luce per il 2000 », in Il Gazzettino, 9 septembre 1998 ; puis in Id., Scritti e parlati, op. cit., p. 32 : « È destino umano abitare il mondo, ma è anche destino sognarne un altro $[\ldots] »$.

13. J. Chabot, La Provence de Giono, op. cit., p. 36.

14. «Un canto d'addio per una Liguria che entra nell'Erebo », F. Biamonti, « Le folate di Vento largo ", in Id., Scritti e parlati, op. cit., p. 80.

15. Id., Vent largue, op. cit., p. 16.

16. « È la civiltà dell'ulivo. Una splendida civiltà. Duemila anni fa i greci ci insegnarono a innestare l'ulivo sul leccio. Oggi, dopo duemila anni, questa società è morta e con lei sono morte le sue comunità. Era una società dolcissima. Qualunque persona con poco più di cinquecento alberi di ulivo poteva vivere bene. Questo ora non è più possibile: gli uliveti rimasti sono quasi tutti abbandonati, la gente di oggi è sola, snaturata. Si sopravvive con le floricolture, le serre hanno sostituito gli uliveti e i più sfortunati sono costretti a fare i camerieri a Montecarlo. La civiltà dell'ulivo è morta ma nessun'altra l'ha sostituita ", Id., " Non c'è più pace tra gli ulivi », interview réalisée par Antonio Troiano, in Corriere della Sera, 31 mars 1991.

17. Cf. A. Malraux, Les noyers de l'Altenburg, Paris, Gallimard, 1948, p. 291. Biamonti cite souvent cette phrase.

18. «[...] non è nemmeno un albero, ma un sogno d'albero ", F. Biamonti, « [sans titre] », in Mario Novaro tra poesia e cultura, Actes de Colloque, Firenze, Le Monnier, 1998 ; puis in Id., Scritti e parlati, op. cit., p. 44.

19. F. Braudel, La Méditerranée. L'espace et l'histoire, Paris, Flammarion, 1977, p. 23.

20. «[...] la zona più a nord dell'uliveto e [...] del palmeto mediterraneo : era, diciamo così, la riva settentrionale di una civiltà antichissima e che si era commista al territorio da sembrare spontanea », F. Biamonti, « [sans titre] », Corrado Ramella (dir.), in Il Gabbiano, 1997, p. 3.

21. J. Giono, Noé, Paris, Éditions de la Table Ronde, 1947, p. 56.

22. F. Biamonti, "Antico! Così lo chiama Montale. Il Mediterraneo è antico e tragico ", in In Europa, 27, 3 août 1991 ; puis in Id., Scritti e parlati, op. cit., p. 122.

23. J. Giono, Manosque-des Plateaux, suivi de Poème de l'olive, Paris, Gallimard, 1998, p. 97.

24. Concernant l'image de la Provence et l'usage de la langue provençale dans les romans de Biamonti, nous renvoyons le lecteur à M. Grassano, " "D'uno lengo vieio que se vôl pas cala...". Francesco Biamonti e il mito provenzale ", in Italica, 94, 3 (2017), pp. 546-562.

25. «Il modello di sviluppo è stato quello delle seconde case, che ha prodotto un fenomeno di conurbazione costiera il cui risultato è la completa saldatura, senza soluzione di continuità, di 
interi tratti di costa », B. Gabrielli, « La dilapidazione del territorio », in La Liguria, Antonio Gibelli et Paride Rugafiori (dir.), Torino, Einaudi, 1994, p. 792.

26. «Io credo che il compito di una civiltà vera sia quello di mantenere le cose perlomeno in una continuità, non staccare brutalmente dal passato; ma siccome tutto è destinato ad andare a morire, che almeno tutto muoia dolcemente. Qui, invece, tutto muore brutalmente : gettano asfalto, erigono palazzi, distruggono il mare, assediano il cimitero di case, distruggono i palmeti... anche distruzioni perfettamente inutili ! », F. Biamonti, « [sans titre] », Corrado Ramella (dir.), in Il Gabbiano, 1997, p. 3.

27. J. Chabot, La Provence de Giono, op. cit., p. 54.

28. J. Giono, Voyage en Italie, Paris, Gallimard, 1953, p. 12.

29. Id., Arcadie... Arcadie..., précédé de La pierre, Gallimard, Paris 2001, p. 122.

30. F. Biamonti, Les paroles la nuit, op. cit., p. 104.

31. "Ora quando io sento qualunque scrittore o interpello qualunque amico che viene qui, mi sento dire: Che cos'è questa Liguria, questa striscia maleodorante di benzina con una selva di case che la circondano ? Un mare che non si vede nemmeno più, di cui non si sente più il respiro, e un entroterra abbandonato ? Ci troviamo davanti a due degradi : uno per un'intensità cancerosa di vita e l'altro per un abbandono lento della vita. / [...] La grande distruzione è avvenuta negli anni '60 e '70, ma è avvenuta in tutto il mondo, è avvenuta in Francia, in Italia, sulla Costa Azzurra, dove non c'è più niente che non sia falso, è avvenuta sommamente a Monaco; e quindi non vado a imputare agli uomini di Bordighera la colpa di una smania frenetica che ha preso tutto il mondo », Id., « [sans titre] », Corrado Ramella (dir.), in Il Gabbiano, 1997, p. 3.

32. G. Bachelard, L'air et les songes. Essai sur l'imagination du mouvement, Paris, José Corti, 1992, p. 18.

33. F. Biamonti, «Biamonti, l'anti-estate », interview réalisée par Antonella Viale, in Il Secolo XIX, 4 août 1996, p. 9 : « [...] l'ancestralità e la sacralità della vita sono ancora valori quotidiani ».

34. C. Baudelaire, «Le cygne », in Id., Les Fleurs du Mal, édition de 1861, texte présenté, établi et annoté par Claude Pichois, Paris, Gallimard, 1996, p. 119.

35. Pour une étude de l'œuvre de Biamonti et celle de Giono à travers la thématique de la frontière voir M. Marengo, "Sguardi letterari sulle "terre di frontiera" : le rappresentazioni delle Alpi sud-occidentali nelle opere di Francesco Biamonti e Jean Giono, in Intemelion », 22 (2016), pp. 89-104.

\section{ABSTRACTS}

Cet article analyse l'influence que l'image de la Provence proposée par Jean Giono a eue sur la représentation du territoire de l'extrême Ponant ligurien dans les romans de Francesco Biamonti (1928-2001). L'écrivain italien ne peut qu'éprouver une grande fascination pour l'œuvre de l'auteur de Manosque: il se rend compte que les transformations sociales, économiques et territoriales subies par la Provence depuis la deuxième moitié du XIXème siècle et dénoncées par Giono avec une force extraordinaire à partir des années vingt sont également celles qui ont bouleversé sa Ligurie, cinquante ans plus tard. Exactement comme la Provence de Giono, la Ligurie de Biamonti est décrite sous son double aspect: d'une part la côte bétonnée, qui est devenue le symbole d'une modernité immorale et brutale ; d'autre part l'arrière-pays qui, même 
à l'abandon, garde encore les traces de l'ancienne "civilisation de l'olivier » et du paysage originaire.

L'articolo studia l'influenza che l'immagine della Provenza di Jean Giono ha avuto sulla rappresentazione del territorio dell'estremo Ponente ligure nei romanzi di Francesco Biamonti (1928-2001). Lo scrittore italiano non può che provare un grande fascino per l'opera dell'autore di Manosque. Si rende conto, infatti, che le trasformazioni sociali, economiche e territoriali subite dalla Provenza dalla metà del XIX secolo e denunciate da Giono con una forza straordinaria già a partire dagli anni Venti sono le stesse che cinquant'anni più tardi hanno sconvolto la Liguria. Esattamente come la Provenza di Giono, la Liguria di Biamonti è divisa in due: da un lato la costa cementificata, simbolo di una modernità immorale e brutale; dall'altro lato un entroterra che, anche se in stato di abbandono, conserva ancora le tracce dell'antica «civiltà dell'ulivo » e del paesaggio originario.

\section{INDEX}

Mots-clés: Biamonti, Giono, Ligurie, Provence, territoire

\section{AUTHOR}

\section{MATTEO GRASSANO}

Université Côte d'Azur, LIRCES

Matteo Grassano est docteur en Langue, Littérature et Civilisation Italiennes (Université Côte d'Azur, cotutelle internationale avec l'Università degli Studi di Pavia). Ses recherches scientifiques portent sur la littérature et sur l'histoire de la langue italienne du XIXème et du XXème siècle. Il est l'auteur de nombreux articles et d'une monographie intitulée La prosa parlata. Percorsi linguistici nell'opera di Edmondo De Amicis (Franco Angeli, Milano 2018). 